\title{
WEIGHTED SUM RATE MAXIMIZATION FOR COGNITIVE MISO BROADCAST CHANNEL: LARGE SYSTEM ANALYSIS
}

\author{
YuanYuan He and Subhrakanti Dey \\ Department of Electrical and Electronic Engineering \\ University of Melbourne, Vic. 3010, Australia
}

\begin{abstract}
This paper considers the ergodic weighted sum rate (WSR) maximization problem for an underlay cognitive radio MISO broadcast channel, where a secondary network, consisting of a base-station with $M$ transmit antennas and $K$ single-antenna secondary users (SUs), is allowed to share the same spectrum with a primary user (PU), under an average transmit sum power (ATTP) constraint $P_{a v}$ and an average interference power (AIP) constraint on the PU. We show that the ATTP constraint is always active, and as $P_{a v} \rightarrow \infty$, the ergodic WSR approaches infinity similar to the conventional non-CR network case. A low-complexity suboptimal beamforming scheme (called partially-projected regularized zero-forcing beamforming 'PP-RZFBF') with a closed-form beamformer is proposed. Due to the non-convexity of PP-RZFBF scheme, a large system analysis is conducted in the limit as $M$ and $K$ approach infinity with a fixed finite ratio $r=\frac{K}{M}$. We derive deterministic limiting approximations for the PP-RZFBF problem which enables us to determine asymptotically optimal beamformers for PP-RZFBF. Numerical simulations illustrate that the asymptotically optimal beamformers turn out to be quite effective even for small $M, K$.
\end{abstract}

Index Terms - Broadcast channel, cognitive radio, beamforming, multiple-input single-output, large system analysis.

\section{INTRODUCTION}

Cognitive radio (CR), a promising approach to dramatically increase the spectrum utilization efficiency by allowing unlicensed/secondary users (SUs) to access the spectrum originally licensed to the primary users (PUs), has recently attracted a broad range of research interests. Effectively, three categories of CR network paradigms have been proposed: interweave, overlay, and underlay [1]. In the underlay systems (the focus of this paper), the SU can transmit even when the PU is active, as long as the resulting interference on PU does not excess an acceptable limit. Thus the transmit power of SUs should be controlled properly to achieve the best trade-off between maximizing the secondary throughput and minimizing the interference on PUs.

Under such a scenario, in order to enhance the secondary throughput, well studied MIMO technology can be employed to fully exploit the spectral efficiency of SUs. Throughput maximization of a CR point-to-point MIMO network with multiple PUs has been considered in [2], showing that beamforming is the optimal transmit strategy for the MISO case. In [3], the authors studied the weighted sum rate (WSR) maximization problem for CR Multi-SUs MIMO broadcast channel (BC) with a dirty paper coding (DPC) precoder (motivated by the fact that DPC is the optimal capacity achieving scheme for non-CR MIMO BC [4]). The corresponding non-convex problem can be transformed into an equivalent convex
CR MIMO MAC problem by applying BC-MAC duality. In [5], the authors provided an state-of-art overview of CR MIMO (PP/BS/MAC/ad hoc). Due to high computational complexity of DPC, suboptimal linear precoding techniques (such as beamforming) have become the focus of substantial research activities. The authors of [6] investigated a non-convex WSR maximization problem for a CR multi-user MISO interference channel with linear beamforming transmission scheme and obtained a locally optimum beamformer using an iterative algorithm.

In this paper, we consider the ergodic WSR maximization problem for an underlay CR multi-user MISO broadcast channel (CR-MISO-BC), subject to an average transmit sum power (ATTP) constraint at the secondary base-station (CR-BS) and an average interference power (AIP) constraint at the PU. The system setting is similar to [3], but unlike [3], we utilize a linear transmit beamforming strategy instead of dirty paper coding (DPC). Our main contributions are summarized as follows:

- In our CR-MISO-BC WSR maximization problem, we prove that the ATTP constraint is always active, and as $P_{a v} \rightarrow \infty$, the ergodic WSR approaches infinity. This is different from prior research on the CR network with only a single antenna at the transmitters and receivers (such as [7][8]), where with fixed $Q_{a v}$, as $P_{a v}$ increases, the capacity saturates since only the AIP constraint is satisfied with equality.

- Due to non-convexity, there is no explicit solution for our problem. Although we can numerically find a local optimum using an iterative algorithm similar to [6], it is computationally intensive and not amenable to further analysis. Motivated by this, we derive a low-complexity albeit suboptimal strategy (called 'PP-RZFBF') with a closed-form beamformer, by combining the regularized zero-forcing beamforming (RZFBF) [9] with the subspace projection idea proposed by [2][5][10].

- Designing the PP-RZFBF beamformer involves finding optimal values of the projection control parameter $\beta$ and the regularization parameter $\alpha$. Guided by a similar approach in non-CR networks, such as [11][12][13] (which only has parameter $\alpha$ ), we derive deterministic large system approximations for the PP-RZFBF scheme in the limit as $M$ and $K$ tend to infinity with fixed ratio $r=\frac{K}{M}$. This allows us to derive the asymptotically optimal $\beta, \alpha$ for the PP-RZFBF scheme and also helps us characterize the asymptotic behavior of our CR-MISO-BC system. To the best of our knowledge, large system analysis on CR network has not been addressed in any existing literature.

- We also show that in the large system limit, as $P_{a v} \rightarrow \infty$, the interference on PU caused by the secondary transmission goes to zero asymptotically. 


\section{SYSTEM MODEL AND PROBLEM FORMULATION}

We consider an underlay CR-MISO-BC, as illustrated in Fig.1, where a CR-BS equipped with $M$ transmit antennas communicates with $K$ single-antenna SUs, in the presence of a single-antenna PU. Regardless of the on/off status of the PU, the secondary network is allowed to share the same narrowband spectrum with the PU, as long as the degradation of the received signal quality of PU caused by the transmission of the secondary network does not exceed an acceptable level. All channels involved are i.i.d. and assumed to be flat-fading. For $i=1, \ldots, K$, let $\mathbf{h}_{i} \in \mathbb{C}^{M \times 1}$ and $\mathbf{h}_{0} \in \mathbb{C}^{M \times 1}$ denote the channel vector from the CR-BS to $\mathrm{SU}_{i}$ and $\mathrm{PU}$, respectively, where the elements of each $\mathbf{h}_{j}, \forall j=0,1, \ldots, K$ are assumed to be i.i.d complex Gaussian random variables with zero mean and unit variance. Then, the received signal at each $\mathrm{SU}_{i}$, indicated by $y_{i}$, is given as, for $i=1, \ldots, K$,

$$
y_{i}=\sqrt{p_{i}} \mathbf{h}_{i}^{\mathcal{H}} \mathbf{g}_{i} s_{i}+\sum_{j \neq i}^{K} \sqrt{p_{j}} \mathbf{h}_{i}^{\mathcal{H}} \mathbf{g}_{j} s_{j}+n_{i},
$$

where $n_{i}$ is the additive white Gaussian noise ${ }^{1}$ at the $\mathrm{SU}_{i}$ and $n_{i} \sim \mathcal{C N}(0,1) ; p_{i} \geq 0$ is the transmit signal power for $\mathrm{SU}_{i} ; s_{i}$ denotes the transmit symbol destined to $\mathrm{SU}_{i}$ and is linearly precoded by the transmit beamforming vector $\mathbf{g}_{i} \in \mathbb{C}^{M \times 1}$. Subsequently, the ATTP constraint can be written as $E\left[\sum_{i=1}^{K} p_{i}\left|\mathbf{g}_{i}\right|^{2}\right] \leq P_{a v}$, where $P_{a v}$ is the maximum average transmit power at CR-BS. Let $Q_{a v}$ denote the average interference power limit tolerated by the PU, then the AIP constraint can be written as, $E\left[\sum_{i=1}^{K} p_{i}\left|\mathbf{h}_{0}^{\mathcal{H}} \mathbf{g}_{i}\right|^{2}\right] \leq Q_{a v}$.

Instead of applying dirty paper coding (DPC) at CR-BS (as [3]) or multiuser decoding at receivers to cancel the multiuser interference, each receiver $\mathrm{SU}_{i}(i=1, \ldots, K)$ decodes the transmitted symbol $s_{i}$ by simply treating the multiuser interference as noise [14][15]. Then given full channel state information (CSI), i.e., $\mathbf{H} \triangleq\left\{\mathbf{h}_{1}, \ldots, \mathbf{h}_{K}\right\} \in \mathcal{C}^{M \times K}$ and $\mathbf{h}_{0}$, the signal to interference plus noise ratio (SINR) at each receiver $\mathrm{SU}_{i}$ is given as, $\operatorname{SINR}_{i}=\frac{p_{i}\left|\mathbf{h}_{i}^{\mathcal{H}} \mathbf{g}_{i}\right|^{2}}{1+\sum_{j=1, j \neq i}^{K} p_{j}\left|\mathbf{h}_{i}^{\mathcal{H}} \mathbf{g}_{j}\right|^{2}}$. Therefore, with the assumption of perfect CSI, the WSR maximization problem for CR-MISO-BC, under both an ATTP constraint at CR-BS and an AIP constraint at the PU, can be formulated as,

$$
\begin{aligned}
& \underset{\left\{\mathbf{g}_{i}\right\}_{i=1}^{K},\left\{p_{i} \geq 0\right\}_{i=1}^{K}}{\operatorname{maximize}} R_{\text {sum }}=\sum_{i=1}^{K} w_{i} E\left[\log \left(1+\operatorname{SINR}_{i}\right)\right] \\
& \text { s.t. } E\left[\sum_{i=1}^{K} p_{i}\left|\mathbf{g}_{i}\right|^{2}\right] \leq P_{a v}, E\left[\sum_{i=1}^{K} p_{i}\left|\mathbf{h}_{0}^{\mathcal{H}} \mathbf{g}_{i}\right|^{2}\right] \leq Q_{a v} .
\end{aligned}
$$

where $w_{i}$ is the weight for $\mathbf{S U}_{i}$ and the expectation is taken over $\mathbf{H}, \mathbf{h}_{0}$.

Proposition 1 In Problem (2), given any value of $P_{a v}$ and $Q_{a v}$, the ATTP constraint is always active, and the ergodic WSR $R_{\text {sum }} \rightarrow \infty$, as $P_{a v} \rightarrow \infty$. (Proof. Please See [16])

It is easy to verify that Problem (2) is a non-convex optimization problem. In general, there is no explicit solution for Problem (2). Although we can numerically find a local optimum for Problem (2) by solving its Lagrange dual problem using an iterative algorithm similar to [6], it is computationally complex and it also hinders

\footnotetext{
${ }^{1}$ Here, similar to [2], the additive noise $n_{i}$ at the receiver $\mathrm{SU}_{i}$ is assumed to include any other interferences from outside of the secondary network, such as, the interference from the primary transmitters
}

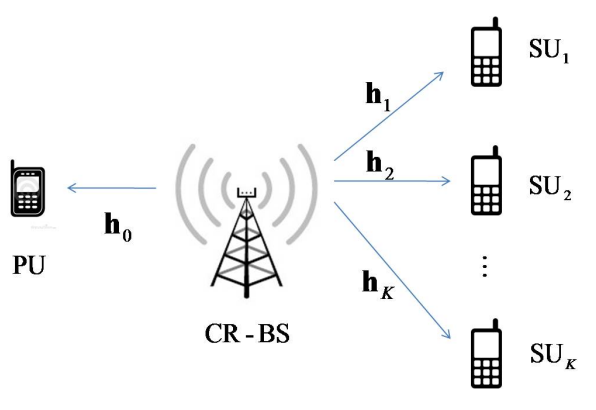

Fig. 1. CR-MISO-BC System Model

further analysis of the beamforming strategy. Next, we will derive a low-complexity albeit suboptimal strategy for Problem (2) with a simple closed-form beamforming solution.

\section{A SUBOPTIMAL BEAMFORMING SCHEME}

Let $\mathbf{G} \triangleq\left\{\mathbf{g}_{1}, \ldots, \mathbf{g}_{K}\right\} \in \mathbb{C}^{M \times K}$ denote the beamforming matrix. Similar to [5], we first look at two extreme cases of Problem (2):

Case I: High $Q_{a v}$ case (large enough to make the AIP constraint inactive). In this scenario, Problem (2) reduces to the conventional non-cognitive MISO-BC WSR optimization problem subject to the ATTP constraint only. A popular, straightforward and effective suboptimal scheme for the conventional MISO-BC is known as zeroforcing beamforming (ZFBF)[9], where the beamforming vectors are designed to satisfy the $\mathrm{ZF}$ criterion (i.e., $\mathbf{h}_{i}^{\mathcal{H}} \mathbf{g}_{j}=0, \forall j \neq i$ ), resulting in complete cancellation of the multiuser interference. One easy choice for the beamforming matrix $\mathbf{G}$ that meets the $\mathrm{ZF}$ criterion is the pseudo-inverse of $\mathbf{H}^{\mathcal{H}}$, i.e., $\mathbf{G}=\mathbf{H}\left(\mathbf{H}^{\mathcal{H}} \mathbf{H}\right)^{-1}$. However, as pointed out by [9], ZFBF has some shortcomings, such as, the inverse of $\mathbf{H}^{\mathcal{H}} \mathbf{H}$ may not exist (for example, when $K>M$, $\left.\operatorname{rank}\left(\mathbf{H}^{\mathcal{H}} \mathbf{H}\right)<K\right)$ and when $K=M$, the sum-rate of ZFBF does not grow linearly with $K$ (or $M$ ). These drawbacks can be improved by adding a regularization term to the ZFBF matrix, given as,

$$
\mathbf{G}=\mathbf{H}\left(\mathbf{H}^{\mathcal{H}} \mathbf{H}+\alpha \mathbf{I}\right)^{-1} \stackrel{(a)}{=}\left(\mathbf{H H}^{\mathcal{H}}+\alpha \mathbf{I}\right)^{-1} \mathbf{H},
$$

where $\alpha>0$ is the regularization parameter and controls the amount of multiuser interference; (a) is given by [12]. This matrix is known as regularized zero-forcing beamforming (RZFBF) matrix. As shown by [9], RZFBF can achieve significantly better sum-rate performance than ZFBF, especially at low $P_{a v}$, and with fixed $K$, as $P_{a v} \rightarrow \infty$, the sum-rate of RZFBF approaches that of ZFBF.

Case II: $Q_{a v}=0$ case. In this case, the AIP constraint in Problem (2) becomes $\mathbf{h}_{0}^{\mathcal{H}} \mathbf{g}_{i}=0, \forall i=1, \ldots, K$, which implies any interference on the PU caused by the secondary network is completely removed. Similar to [2][5], we first project $\mathbf{H}$ into the null space of $\mathbf{h}_{0}$, given as

$$
\mathbf{H}_{\perp}=\left(\mathbf{I}-\overline{\mathbf{h}}_{0} \overline{\mathbf{h}}_{0}^{\mathcal{H}}\right) \mathbf{H},
$$

where $\overline{\mathbf{h}}_{0}=\frac{\mathbf{h}_{0}}{\left|\mathbf{h}_{0}\right|}$, and then design the beamforming matrix $G$ based on the projected channel matrix $\mathbf{H}_{\perp}$, so that the constraints can $\mathbf{h}_{0}^{\mathcal{H}} \mathbf{g}_{i}=0, \forall i=1, \ldots, K$ can be met. More specifically, from (4), we have $\mathbf{H}=\mathbf{H}_{\perp}+\overline{\mathbf{h}}_{0} \overline{\mathbf{h}}_{0}^{\mathcal{H}} \mathbf{H}$ and $\mathbf{h}_{0}^{\mathcal{H}} \mathbf{H}_{\perp}=\mathbf{0}$, which gives $\mathbf{H}^{\mathcal{H}} \mathbf{g}_{i}=\mathbf{H}_{\perp}^{\mathcal{H}} \mathbf{g}_{i}, \forall i=1, \ldots, K$. Applying this to (2), Problem (2) again becomes a conventional non-cognitive MISO-BC with channel matrix $\mathbf{H}_{\perp}$, where according to Case I, the suboptimal RZFBF 
scheme can be applied to design the beamforming matrix, given as

$$
\mathbf{G}=\mathbf{H}_{\perp}\left(\mathbf{H}_{\perp}^{\mathcal{H}} \mathbf{H}_{\perp}+\alpha \mathbf{I}\right)^{-1}=\left(\mathbf{H}_{\perp} \mathbf{H}_{\perp}^{\mathcal{H}}+\alpha \mathbf{I}\right)^{-1} \mathbf{H}_{\perp} .
$$

Obviously, from (5), $\mathbf{h}_{0}^{\mathcal{H}} \mathbf{G}=0$, i.e., the constraint $\mathbf{h}_{0}^{\mathcal{H}} \mathbf{g}_{i}=0, \forall i=$ $1, \ldots, K$ is always satisfied.

In both of the above two special cases, the RZFBF scheme is employed to design the beamforming matrix based on a certain form of $\mathbf{H}$ (which preserves a certain orthogonality to $\mathbf{h}_{0}$ ). By comparing (3) and (5), it seems that as $Q_{a v}$ decreases, the amount of projection of $\mathbf{H}$ into the null space of $\mathbf{h}_{0}$ also increases. This motivates a general heuristic suboptimal method for Problem (2), called partially-projected-RZFBF (PP-RZFBF). In this method, similar to [2][5][10], first the secondary channel matrix $\mathbf{H}$ is projected into the null space of a certain subspace of $\mathbf{h}_{0}$, denoted as $\tilde{\mathbf{H}}$ and given as [10]

$$
\tilde{\mathbf{H}}=\left(\mathbf{I}-\beta \overline{\mathbf{h}}_{0} \overline{\mathbf{h}}_{0}^{\mathcal{H}}\right) \mathbf{H},
$$

where $0 \leq \beta \leq 1$ is the projection control parameter. The RZFBF algorithm is then applied to $\tilde{\mathbf{H}}$ to obtain the beamforming matrix, namely,

$$
\mathbf{G}=\tilde{\mathbf{H}}\left(\tilde{\mathbf{H}}^{\mathcal{H}} \tilde{\mathbf{H}}+\alpha \mathbf{I}\right)^{-1}=\left(\tilde{\mathbf{H}} \tilde{\mathbf{H}}^{\mathcal{H}}+\alpha \mathbf{I}\right)^{-1} \tilde{\mathbf{H}}
$$

which gives, $\mathbf{g}_{i}=\left(\tilde{\mathbf{H}} \tilde{\mathbf{H}}^{\mathcal{H}}+\alpha \mathbf{I}\right)^{-1} \tilde{\mathbf{h}}_{i}, \forall i=1, \ldots, K$ where the vector $\tilde{\mathbf{h}}_{i}$ is the $i^{t h}$ column of $\tilde{\mathbf{H}}$. Obviously, $\beta=0$ and $\beta=1$ are corresponding to Case I and Case II, respectively.

Therefore, Problem (2) with a suboptimal PP-RZFBF scheme can be simplified as,

$$
\begin{array}{cl}
\underset{\beta, \alpha,\left\{p_{i}\right\}_{i=1}^{K}}{\operatorname{maximize}} & R_{\mathrm{PP}-\mathrm{RZFBF}}=\sum_{i=1}^{K} E\left[w_{i} \log \left(1+\operatorname{SINR}_{i}\right)\right] \\
\text { s.t. } & E[\phi] \leq P_{a v}, \\
& E[\psi] \leq Q_{a v}, \\
& 0 \leq \beta \leq 1, \quad \alpha>0, p_{i} \geq 0, \forall i=1, \ldots, K .
\end{array}
$$

where $\phi \triangleq \sum_{i=1}^{K} p_{i} \tilde{\mathbf{h}}_{i}^{\mathcal{H}}\left(\tilde{\mathbf{H}} \tilde{\mathbf{H}}^{\mathcal{H}}+\alpha \mathbf{I}\right)^{-2} \tilde{\mathbf{h}}_{i}, \psi \triangleq \sum_{i=1}^{K} p_{i} \mid \mathbf{h}_{0}^{\mathcal{H}}\left(\tilde{\mathbf{H}} \tilde{\mathbf{H}}^{\mathcal{H}}+\right.$ $\alpha \mathbf{I})\left.^{-1} \tilde{\mathbf{h}}_{i}\right|^{2}$ and $\operatorname{SINR}_{i}=\frac{p_{i}\left|\mathbf{h}_{i}^{\mathcal{H}}\left(\tilde{\mathbf{H}} \tilde{\mathbf{H}}^{\mathcal{H}}+\alpha \mathbf{I}\right)^{-1} \tilde{\mathbf{h}}_{i}\right|^{2}}{1+\mathbf{h}_{i}^{\mathcal{H}}\left(\tilde{\mathbf{H}} \tilde{\mathbf{H}}^{\mathcal{H}}+\alpha \mathbf{I}\right)^{-1} \tilde{\mathbf{H}}_{-i} \mathbf{P}_{-i} \tilde{\mathbf{H}}_{-i}^{\mathcal{H}}\left(\tilde{\mathbf{H}} \tilde{\mathbf{H}}^{\mathcal{H}}+\alpha \mathbf{I}\right)^{-1} \mathbf{h}_{i}}$, with $\tilde{\mathbf{H}}_{-i} \triangleq\left\{\tilde{\mathbf{h}}_{1}, \ldots, \tilde{\mathbf{h}}_{i-1}, \tilde{\mathbf{h}}_{i+1}, \ldots, \tilde{\mathbf{h}}_{K}\right\} \in \mathcal{C}^{M \times K-1}$ and $\mathbf{P}_{-i} \triangleq \operatorname{diag}\left(p_{1}, \ldots, p_{i-1}, p_{i+1}, \ldots, p_{K}\right)$. Although the complexity is largely reduced compared to Problem (2), Problem (8) is still a non-convex optimization problem. However, for given $\beta$ and $\alpha$, a locally optimal power allocation scheme can be obtained by using the SCALE algorithm proposed by [17]. Then, one can utilize a two-dimensional exhaustive search method to get the optimal $\beta$ and $\alpha$, with which, a locally optimal PP-RZFBF beamformer can be obtained. Obviously, an exhaustive search over both $\beta$ and $\alpha$ is rather difficult, especially when the searching range for $\alpha$ is from 0 to $\infty$. This motivates us to study the large system case, which enables us to derive simple but asymptotically optimal expressions for $\beta^{*}$ and $\alpha^{*}$.

\section{LARGE SYSTEM ANALYSIS}

In this section, we will investigate the large system approximations of the PP-RZFBF scheme in order to characterize the asymptotical behavior of our CR-MISO-BC system, and derive the asymptotically optimal solutions of Problem (8), in the limit as $M$ and $K$ grow jointly to infinity with a fixed ratio $r=\frac{K}{M}<\infty$ (called the large system limit). We assume that $\frac{1}{M} \mathbf{H H}$ has a uniformly bounded spectral norm for all $M$ and $\max \left(p_{1}, \ldots, p_{K}\right)=\mathcal{O}\left(\frac{1}{K}\right)$. Then, guided by the approach in [11][12][13], we can show in the Theorem below that in the large system limit, $\operatorname{SINR}_{i}, \phi, \psi$ converge almost surely to deterministic values, denoted by $\operatorname{SINR}_{i}^{\infty}, \phi^{\infty}, \psi^{\infty}$, respectively. Later, this will enable us to determine the asymptotically optimal design parameters $\alpha^{*}, \beta^{*}$ for Problem (8).

Theorem 1 As $M \rightarrow \infty, K \rightarrow \infty$ with a fixed and finite $r=\frac{K}{M}$,

$$
\begin{aligned}
& \operatorname{SINR}_{i} \stackrel{a . s .}{\rightarrow} \frac{p_{i} z\left(r, \alpha_{0}\right)^{2}}{\left(1+z\left(r, \alpha_{0}\right)\right)^{2}+\frac{r z\left(r, \alpha_{0}\right)\left(\frac{1}{K} \sum_{j=1}^{K} p_{j}-\frac{p_{i}}{K}\right)}{r+\alpha_{0}\left(1+z\left(r, \alpha_{0}\right)\right)^{2}}}, \\
& \phi \stackrel{a . s .}{\rightarrow} \frac{r z\left(r, \alpha_{0}\right)}{r+\alpha_{0}\left(1+z\left(r, \alpha_{0}\right)\right)^{2}}\left(\frac{1}{K} \sum_{i=1}^{K} p_{i}\right), \\
& \psi \stackrel{\text { a.s. }}{ } \\
& \frac{(1-\beta)^{2} r z\left(r, \alpha_{0}\right)\left(\frac{1}{K} \sum_{i=1}^{K} p_{i}\right)}{\left(1+\left(\beta^{2}-2 \beta\right)\left(1-\alpha_{0} z\left(r, \alpha_{0}\right)\right)^{2}\left(r+\alpha_{0}\left(1+z\left(r, \alpha_{0}\right)\right)^{2}\right)\right.},
\end{aligned}
$$

where $z\left(r, \alpha_{0}\right)=\frac{1}{2}\left[\sqrt{\frac{(1-r)^{2}}{\alpha_{0}^{2}}+\frac{2(1+r)}{\alpha_{0}}+1}+\frac{1-r}{\alpha_{0}}-1\right]$ and $\alpha_{0}=$ $\frac{\alpha}{M}$. (Proof. Please See [16])

From Theorem 1, it is seen that $\operatorname{SINR}_{i}^{\infty}, \phi^{\infty}, \psi^{\infty}$ are all deterministic quantities and do not depend on any $\operatorname{CSIT}\left(\mathbf{H}, \mathrm{h}_{0}\right)$, which implies that in the large system limit, CSIT information is no longer required. Note that with assumption $\max \left(p_{1}, \ldots, p_{K}\right)=\mathcal{O}\left(\frac{1}{K}\right)$, the term $\frac{p_{i}}{K}$ in (9a) can be omitted since $\frac{p_{i}}{K} \ll \frac{1}{K} \sum_{j=1}^{K} p_{j}$ as $K \rightarrow \infty$ and without $\frac{p_{i}}{K}$ the convergence in (9a) still holds true. Let $\tau\left(r, \alpha_{0}\right) \triangleq \frac{r z\left(r, \alpha_{0}\right)}{r+\alpha_{0}\left(1+z\left(r, \alpha_{0}\right)\right)^{2}}, \rho\left(r, \alpha_{0}, \beta\right) \triangleq \frac{(1-\beta)^{2}}{\left(1+\left(\beta^{2}-2 \beta\right)\left(1-\alpha_{0} z\left(r, \alpha_{0}\right)\right)\right)^{2}}$ and $v_{0} \triangleq \frac{z\left(r, \alpha_{0}\right)^{2}}{\left(1+z\left(r, \alpha_{0}\right)\right)^{2}+\tau\left(r, \alpha_{0}\right) \frac{1}{K} \sum_{j=1}^{K} p_{j}}$. Then, $\operatorname{SINR}_{i}^{\infty}, \phi^{\infty}, \psi^{\infty}$ can be rewritten as $\operatorname{SINR}_{i}^{\infty}=p_{i} v_{0}, \phi^{\infty}=\tau\left(r, \alpha_{0}\right) \frac{1}{K} \sum_{i=1}^{K} p_{j}$, and $\psi^{\infty}=\rho\left(r, \alpha_{0}, \beta\right) \tau\left(r, \alpha_{0}\right) \frac{1}{K} \sum_{i=1}^{K} p_{j}$, respectively . Therefore, in the large system limit, the Problem (8) becomes,

$$
\begin{aligned}
\underset{0 \leq \beta \leq 1, \alpha_{0}>0, p_{i} \geq 0, \forall i=1, \ldots, K}{\operatorname{maximize}} & R_{\mathrm{PP}-\mathrm{RZFBF}}^{\infty} & =\sum_{i=1}^{K} w_{i} \log \left(1+p_{i} v_{0}\right) \\
\text { s.t. } & \frac{1}{K} \sum_{i=1}^{K} p_{i} & =\bar{P}
\end{aligned}
$$

where

$\bar{P}= \begin{cases}\min \left\{\frac{P_{a v}}{\tau\left(r, \alpha_{0}\right)}, \frac{Q_{a v}}{\rho\left(r, \alpha_{0}, \beta\right) \tau\left(r, \alpha_{0}\right)}\right\}, & \text { when } \rho\left(r, \alpha_{0}, \beta\right) \neq 0 \\ \frac{P_{a v}}{\tau\left(r, \alpha_{0}\right)}, & \text { when } \rho\left(r, \alpha_{0}, \beta\right)=0 .\end{cases}$

Applying the power constraint of Problem (10) into the definition of $v_{0}$, we have $v_{0}=\frac{z\left(r, \alpha_{0}\right)^{2}}{\left(1+z\left(r, \alpha_{0}\right)\right)^{2}+\tau\left(r, \alpha_{0}\right) \bar{P}}$, which is the same for all $\mathrm{SU}_{i}, i=1, \ldots, K$.

Next, we will find the asymptotically optimal design parameters by solving the optimization problem (10).

Theorem 2 As $M, K \rightarrow \infty$ with a bounded ratio $r$, the asymptotically optimal regularization parameter $\alpha_{0}$, asymptotically optimal projection control parameter $\beta$ as well as the asymptotically optimal power allocation for Problem (8) are given, respectively, as:

$$
\alpha_{0}^{*}=\frac{r}{P_{a v}}
$$


- When $0 \leq \sqrt{\frac{Q_{a v}}{P_{a v}}}<1$, we have $\phi_{1} \leq \beta^{*} \leq 1$;

- When $1 \leq \sqrt{\frac{Q_{a v}}{P_{a v}}} \leq \frac{1}{2} \frac{1}{\sqrt{\alpha_{0}^{*} z\left(r, \alpha_{0}^{*}\right)\left(1-\alpha_{0}^{*} z\left(r, \alpha_{0}^{*}\right)\right)}}$, we have,

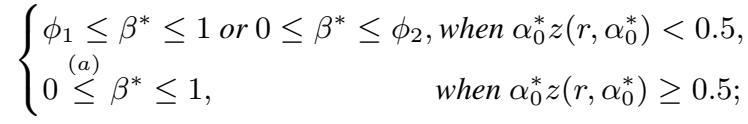

- when $\sqrt{\frac{Q_{a v}}{P_{a v}}}>\frac{1}{2} \frac{1}{\sqrt{\alpha_{0}^{*} z\left(r, \alpha_{0}^{*}\right)\left(1-\alpha_{0}^{*} z\left(r, \alpha_{0}^{*}\right)\right)}}, 0 \leq \beta^{*} \leq 1$.

where in (a), the equality could only happen when $\sqrt{\frac{Q_{a v}}{P_{a v}}}=$ $1 ; \phi_{1} \triangleq J\left(\alpha_{0}^{*}, z\left(r, \alpha_{0}^{*}\right), t\right)-\frac{1}{2 t\left(1-\alpha_{0}^{*} z\left(r, \alpha_{0}^{*}\right)\right)}+1$ and $\phi_{2} \triangleq$ $-J\left(\alpha_{0}^{*}, z\left(r, \alpha_{0}^{*}\right), t\right)-\frac{1}{2 t\left(1-\alpha_{0}^{*} z\left(r, \alpha_{0}^{*}\right)\right)}+1$ with $J\left(\alpha_{0}^{*}, z\left(r, \alpha_{0}^{*}\right), t\right)$ $\triangleq \sqrt{1-\frac{1}{1-\alpha_{0}^{*} z\left(r, \alpha_{0}^{*}\right)}+\frac{1}{4 t^{2}\left(1-\alpha_{0}^{*} z\left(r, \alpha_{0}^{*}\right)\right)^{2}}}$, and $t=\sqrt{\frac{Q_{a v}}{P_{a v}}}$.

$\left\{p_{i}^{*}\right\}_{i=1}^{K}$ is given by the conventional water-filling, i.e.,

$$
p_{i}^{*}=\left[\frac{w_{i}}{\lambda_{\infty}}-\frac{1}{v_{0}}\right]^{+}, \forall i=1, \ldots, K
$$

where $\lambda_{\infty}$ is the Lagrange multiplier determined by $\frac{1}{K} \sum_{i=1}^{K} p_{i}=$ $\bar{P}$. Without loss of generality, assuming $w_{1} \geq \cdots \geq w_{K}$, from (13), we obtain $p_{1} \geq \cdots \geq p_{K}$. Assuming there is $\bar{K}$ non-zero powers and applying (13) into the power constraint $\frac{1}{K} \sum_{i=1}^{K} p_{i}=\bar{P}$, we can obtain $\lambda_{\infty}=\frac{\frac{1}{K} \sum_{i=1}^{\bar{K}} w_{i}}{\frac{\bar{K}}{K} \frac{1}{v_{0}}+\bar{P}}$. (Proof. Please See [16])

Based on Theorem 2, we have

$$
\begin{aligned}
& R_{\mathrm{PP}-\mathrm{RZFBF}}^{\infty}=\left(\sum_{i=1}^{\bar{K}} w_{i}\right) \log \left(\frac{\bar{K}}{K}+\bar{P} v_{0}\right) \\
& +\sum_{i=1}^{\bar{K}} w_{i} \log w_{i}-\left(\sum_{i=1}^{\bar{K}} w_{i}\right) \log \left(\frac{1}{K} \sum_{i=1}^{\bar{K}} w_{i}\right)
\end{aligned}
$$

and the optimal $\bar{P}$ is always $\bar{P}=\frac{P_{a v}}{\tau\left(r, \alpha_{0}^{*}\right)}$. Note that in the case of $w_{1}=\cdots=w_{K}=w$, we have equal power allocation for each SUs, i.e., $p_{1}=\cdots=p_{K}=\bar{P}$, and the corresponding $R_{\mathrm{PP}-\mathrm{RZFBF}}^{\infty}=$ $K w \log \left(1+\bar{P} v_{0}\right)$.

Proposition 2 In the large system limit, for any given $r$, as $P_{a v} \rightarrow$ $\infty$, we have $\alpha_{0}^{*} \rightarrow 0$ and $\beta^{*} \rightarrow 1$. (Proof. Please See [16])

Proposition 2 implies that in the large system limit, as $P_{a v} \rightarrow \infty$, the secondary channel partial projection $\tilde{\mathbf{H}}$ is asymptotically orthogonal to the primary channel $\mathbf{h}_{0}$ and the interference on PU caused by the secondary transmission is asymptotically cancelled.

\section{NUMERICAL RESULTS}

In Fig.2, we compare the ergodic WSR between the optimal PPRZFBF, obtained via an exhaustive search over all possible $\alpha$ and $\beta$, and the PP-RZFBF directly applied with the asymptotically optimal $\alpha^{*}$ and $\beta^{*}$ obtained from large system analysis. We call it as 'PPRZFBF-ABF', and since $\beta^{*}$ is given by a range of values, a one dimensional exhaustive search over all possible $\beta^{*}$ is also required but it is computationally less complex than the optimal PP-RZFBF case. These results are obtained for a finite system with $K=M=3$ for different $Q_{a v}$. Both cases have been averaged over 10000 independent channel realization. It can be observed from Fig.2 that, for each $Q_{a v}$, these two performances are very close to each other. Thus even

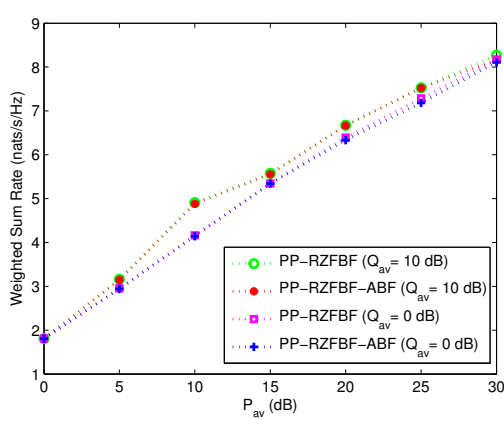

Fig. 2. Ergodic WSR performance comparison between PP-RZFBF and PP-RZFBF with the asymptotically optimal beamformer (PPRZFBF-ABF) for $K=M=3$.

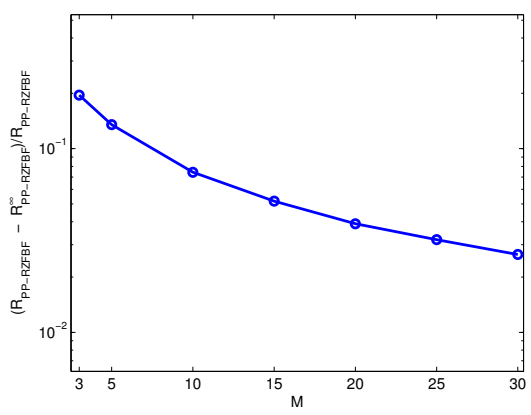

Fig. 3. The difference between the limiting approximation for WSR of PP-RZFBF and the corresponding optimal PP-RZFBF scheme for a fixed $P_{a v}=10 \mathrm{~dB}, Q_{a v}=10 \mathrm{~dB}$ with $M=K$.

for small values of $K, M$, the asymptotically optimal $\alpha^{*}$ and $\beta^{*}$ can provide pretty accurate approximations. Fig. 3 depicts the difference between large system analysis based deterministic approximation of WSR of PP-RZFBF, i.e., $R_{\mathrm{PP}-\mathrm{RZFBF}}^{\infty}$ and the $R_{\mathrm{PP}-\mathrm{RZFBF}}$ of the optimal PP-RZFBF-ABF scheme for a fixed $P_{a v}=10 \mathrm{~dB}, Q_{a v}=10 \mathrm{~dB}$ with $M=K$. From Fig.3, we can see that $R_{\mathrm{PP}-\mathrm{RZFBF}}^{\infty}$ becomes more accurate as $M$ increases.

\section{CONCLUSIONS}

In this paper, we consider the optimal transmit beamforming scheme for an underlay CR-MISO-BC ergodic WSR maximization problem, subject to an ATTP constraint at the CR-BS and an AIP constraint on the PU. In order to further analyze the beamforming technique, we propose a low-complexity suboptimal beamforming scheme (called partially-projected regularized zero-forcing beamforming 'PP-RZFBF') with a closed-form beamformer. A large system analysis is then applied to derive deterministic approximations for PP-RZFBF scheme, based on which, asymptotically optimal values for the parameters $\beta$ and $\alpha$ for PP-RZFBF can be obtained. Numerical simulations confirm the accuracy of asymptomatic optimal $\beta$ and $\alpha$ for finite-sized systems and illustrate that the asymptotic expression of WSR approximates the PP-RZFBF behavior extremely well for large $M$ with $r=1$. 


\section{REFERENCES}

[1] I. Maric A. Goldsmith, S.A. Jafar and S. Srinivasa, "Breaking spectrum gridlock with cognitive radios: an information theoretic perspective," Proc. of the IEEE, vol. 97, no. 5, pp. 894-914, May 2009.

[2] R. Zhang and Y. Liang, "Exploiting multi-antennas for opportunistic spectrum sharing in cognitive radio networks," IEEE Journal of Selected Topics in Signal Process., vol. 2, no. 1, pp. 88-102, Feb. 2008.

[3] Y. Xin L. Zhang and Y. Liang, "Weighted sum rate optimization for cognitive radio mimo broadcast channels," IEEE Trans. Wireless Commun., vol. 8, no. 6, pp. 2950-2959, Jun. 2009.

[4] G. Caire and S. Shamai, "On the achievable throughput of a multiantenna gaussian broadcast channel," IEEE Trans. Inform. Theory, vol. 49, no. 7, pp. 1691-1706, Jul. 2003.

[5] Y. Liang R. Zhang and S. Cui, "Dynamic resource allocation in cognitive radio networks," IEEE Signal Process. Mag., vol. 27, no. 3, pp. 102-114, May 2010.

[6] I. Ghauri L. Gallo, F. Negro and D.T.M. Slock, "Weighted sum rate maximization in the underlay cognitive miso interference channel," in Proc. IEEE PIMRC, 2011, pp. 661-665.

[7] A. Ghasemi and E. S. Sousa, "Fundamental limits of spectrumsharing in fading environments," IEEE Trans. Wireless Commun., vol. 6, no. 2, pp. 649-658, Feb. 2007.

[8] Y. Xin L. Zhang and Y. Liang, "Optimal power allocation for multiple access channels in cognitive radio networks," in Proc. IEEE VTC Spring, 2008, pp. 1549-1553.

[9] B. M. Hochwald C. B. Peel and A. L. Swindlehurst, "A vector-perturbation technique for near-capacity multiantenna multiuser communication-part i: channel inversion and regularization," IEEE Trans. Commun., vol. 53, no. 1, pp. 195-202, Jan. 2005.

[10] Y. C. Liang K. Jitvanichphaibool and R. Zhang, "Beamforming and power control for multi-antenna cognitive two-way relaying," in Proc. IEEE WCNC, 2009, pp. 1-6.

[11] M. Debbah S. Wagner, R. Couillet and D. T. M. Slock, "Large system analysis of linear precoding in correlated miso broadcast channels under limited feedback," arXiv:0906.3682v5, Feb. 2012

[12] R. Muharar V.K. Nguyen and J. Evans, "Multiuser transmit beamforming via regularized channel inversion: A large system analysis," http://goo.gl/Ecafy.

[13] R. Muharar and J. Evans, "Downlink beamforming with transmit-side channel correlation: A large system analysis," in Proc. IEEE ICC, 2011, pp. 1-5.

[14] M. Kobayashi and G. Caire, "Joint beamforming and scheduling for a multi-antenna downlink with imperfect transmitter channel knowledge," IEEE Journal on Selected Areas in Commun., vol. 25, no. 7, pp. 1468-1477, Sep. 2007.

[15] T. Yoo and A. Goldsmith, "On the optimality of multiantenna broadcast scheduling using zero-forcing beamforming," IEEE Journal on Selected Areas in Commun., vol. 24, no. 3, pp. 528541, Mar. 2006.

[16] Y. Y. He and S. Dey, "Wsr-proofs.pdf," URL: https://sites.google.com/site/zhyfutopia/Home/WSRproofs.pdf.
[17] J. Papandriopoulos and J.S. Evans, "Scale: A low-complexity distributed protocol for spectrum balancing in multiuser dsl networks," IEEE Trans. Inform. Theory, vol. 55, no. 8, pp. 3711-3724, Aug. 2009. 